\title{
Alternative twisted tensor products and Cayley algebras
}

\author{
Helena Albuquerque* \\ Departamento de Matemática, Universidade de Coimbra, \\ 3001-454 Coimbra, Portugal \\ e-mail: lena@mat.uc.pt \\ Florin Panaite ${ }^{\dagger}$ \\ Institute of Mathematics of the Romanian Academy \\ PO-Box 1-764, RO-014700 Bucharest, Romania \\ e-mail: Florin.Panaite@imar.ro
}

\begin{abstract}
We introduce what we call alternative twisted tensor products for not necessarily associative algebras, as a common generalization of several different constructions: the CayleyDickson process, the Clifford process and the twisted tensor product of two associative algebras, one of them being commutative. We show that some very basic facts concerning the Cayley-Dickson process (the equivalence between the two different formulations of it and the lifting of the involution) are particular cases of general results about alternative twisted tensor products of algebras. As a class of examples of alternative twisted tensor products, we introduce a tripling process for an algebra endowed with a strong involution, containing the Cayley-Dickson doubling as a subalgebra and sharing some of its basic properties.
\end{abstract}

Keywords: twisted tensor products; Cayley algebras

2000 Mathematics Subject Classification: 17A01

\section{Introduction}

If $A$ and $B$ are associative algebras and $R: B \otimes A \rightarrow A \otimes B$ is a linear map satisfying a certain list of axioms, then $A \otimes B$ becomes an associative algebra with a multiplication defined in terms of $R$ and the multiplications of $A$ and $B$. This construction appeared in several contexts and under different names. Following [9] we call such an $R$ a twisting map and the algebra structure on $A \otimes B$ afforded by it the twisted tensor product of $A$ and $B$ and denote it by $A \otimes_{R} B$ (if $R$ is the usual flip map then $A \otimes_{R} B$ coincides with the usual tensor product of algebras). The twisted tensor product of associative algebras can be regarded as a representative for the cartesian product of noncommutative spaces, better suited than the ordinary tensor product, see [9], 11], 12] for a detailed discussion and references. Examples of twisted tensor products are provided by classical ring theory (crossed products with trivial cocycles, Ore extensions with trivial derivations), Hopf algebra theory (smash products, diagonal crossed products as in [8], [10]), noncommutative geometry etc.

\footnotetext{
${ }^{*}$ The first author was partially supported by the Centre for Mathematics of the University of Coimbra (CMUC)

${ }^{\dagger}$ Research started while the second author was visiting the Centre for Mathematics of the University of Coimbra (CMUC) supported by a postdoctoral fellowship offered by CMUC, and finished while this author was a member of the CNCSIS project "Hopf algebras, cyclic homology and monoidal categories", contract nr. 560/2009, CNCSIS code $I D_{-} 69$.
} 
An important feature of this construction is that it allows to build new algebra structures starting with two given algebras. From this point of view, and also having in mind the emerging nonassociative geometry (cf. [1], 6], [13), regarded as a further extension of noncommutative geometry, with the "coordinate algebra" allowed to be nonassociative, it is natural to try to consider analogues of twisted tensor products for more general classes of algebras than the associative ones. It turns out that it is straightforward to do this for the so-called quasialgebras as named in [3], that is algebras in monoidal categories (since the associativity constraints are allowed to be nontrivial, the class of quasialgebras contains various classes of nonassociative algebras, for instance the octonions and the other Cayley algebras, cf. [3]). Twisted tensor products of certain quasialgebras have been studied by the authors in [5].

In this paper we will introduce a different kind of twisted tensor product for nonassociative algebras, having as motivating example the Cayley-Dickson process. We recall that if $B$ is an algebra endowed with an involution $\sigma: B \rightarrow B$ and $q$ is a nonzero element in the base field $K$, the Cayley-Dickson algebra $\bar{B}(q)$ is an algebra structure on $B \oplus B$, whose elements are written uniquely as $a+v b$, with $a, b \in B$, where $v$ is a notational device with $v^{2}=q 1$, and whose multiplication is defined by

$$
(a+v b)(c+v d)=(a c+q d \sigma(b))+v(\sigma(a) d+c b), \quad \forall a, b, c, d \in B .
$$

If we denote by $C(K, q)$ the 2-dimensional algebra $C(K, q):=K[v] /\left(v^{2}-q\right)$, it is clear that $\bar{B}(q)$ may be identified as vector space with $C(K, q) \otimes B$, and the multiplication of $\bar{B}(q)$ depends somehow on the multiplications of $C(K, q)$ and $B$. Our original purpose was to make exact sense of this claim; more precisely, we wanted to express $\bar{B}(q)$ as some sort of twisted tensor product between $C(K, q)$ and $B$.

The general construction that we introduce, with $\bar{B}(q)$ as the motivating example, is called alternative twisted tensor product between two nonassociative algebras $A$ and $B$ and is defined by means of a so-called alternative twisting map $R: B \otimes A \rightarrow A \otimes B$, which is a linear map satisfying some axioms resembling the ones for a twisting map. We emphasize that a twisting map between two associative algebras $A$ and $B$ in general is not an alternative twisting map. However, if $B$ is moreover commutative, the two concepts coincide.

Our main results (Theorems 2.1 and 3.1) show that some basic features of the Cayley-Dickson process (the fact that it admits two different but equivalent descriptions and the fact that the involution $\sigma$ can be lifted to an involution on $\bar{B}(q)$ ), which apparently are very peculiar to it, are actually particular cases of general results about alternative twisted tensor products of algebras. This kind of phenomenon appeared also in [11, where it was shown that various results from Hopf algebra theory are actually particular cases of general results about twisted tensor products of associative algebras.

As a class of examples of alternative twisted tensor products, we introduce a sort of tripling process: if $B$ is an algebra endowed with a strong involution $\sigma$ and $q, r$ are nonzero scalars, we construct an algebra $\bar{B}(q, r)$, whose dimension is $3 \cdot \operatorname{dim}(B)$, containing the Cayley-Dickson algebras $\bar{B}(q)$ and $\bar{B}(r)$ as subalgebras. Unlike $\bar{B}(q)$ (which is alternative when $B$ is associative), the algebras $\bar{B}(q, r)$ are never alternative algebras. But, exactly as for Cayley algebras, $\bar{B}(q, r)$ is always power-associative, it is flexible if and only if $B$ is flexible and if the norm on $B$ is nondegenerate then so is the norm on $\bar{B}(q, r)$. 


\section{Alternative twisted tensor products of algebras}

In what follows, by "algebra" we will mean a not necessarily associative algebra with unit over a field $K$ (of characteristic $\neq 2$ if necessary); all algebra maps are assumed to be unital. For an algebra $A$ we will denote its multiplication by $\mu_{A}$ and its unit by $1_{A}$. All algebras that will appear will be considered together with a given and fixed decomposition as a direct sum of vector spaces $A=K \cdot 1_{A} \oplus A_{0}$ (our constructions will depend on the decomposition). If $q \in K, q \neq 0$, the decomposition for the algebra $C(K, q):=K[v] /\left(v^{2}-q\right)$ will always be $C(K, q)=K \cdot 1 \oplus K \cdot v$. If $V, W$ are vector spaces, we denote by $\tau_{V, W}: V \otimes W \rightarrow W \otimes V$ the flip map $v \otimes w \mapsto w \otimes v$.

We recall from [9], [16] the construction of the twisted tensor product of associative algebras. Let $A$ and $B$ be two associative algebras and $R: B \otimes A \rightarrow A \otimes B$ a linear map, with Sweedlertype notation $R(b \otimes a)=a_{R} \otimes b_{R}$, for $a \in A, b \in B$. Then $R$ is called twisting map if the following conditions are satisfied (we denote by $r$ another copy of $R$ ):

$$
\begin{aligned}
& R\left(1_{B} \otimes a\right)=a \otimes 1_{B}, \quad R\left(b \otimes 1_{A}\right)=1_{A} \otimes b, \\
& R\left(b \otimes a a^{\prime}\right)=a_{R} a_{r}^{\prime} \otimes\left(b_{R}\right)_{r}, \\
& R\left(b b^{\prime} \otimes a\right)=\left(a_{R}\right)_{r} \otimes b_{r} b_{R}^{\prime},
\end{aligned}
$$

for all $a, a^{\prime} \in A$ and $b, b^{\prime} \in B$. If we introduce a multiplication on $A \otimes B$, by

$$
(a \otimes b)\left(a^{\prime} \otimes b^{\prime}\right)=a a_{R}^{\prime} \otimes b_{R} b^{\prime}, \quad \forall a, a^{\prime} \in A, b, b^{\prime} \in B,
$$

then this multiplication is associative with unit $1_{A} \otimes 1_{B}$. This algebra structure on $A \otimes B$ is called the twisted tensor product afforded by $R$ and is denoted by $A \otimes_{R} B$.

Let $A$ and $B$ be two algebras and $R: B \otimes A \rightarrow A \otimes B$ a linear map, with Sweedler-type notation $R(b \otimes a)=a_{R} \otimes b_{R}$, for $a \in A, b \in B$. Assume that the following conditions are satisfied (we denote by $r$ another copy of $R$ ):

$$
\begin{aligned}
& R\left(1_{B} \otimes a\right)=a \otimes 1_{B}, \quad R\left(b \otimes 1_{A}\right)=1_{A} \otimes b, \quad \forall a \in A, b \in B, \\
& R\left(b \otimes a a^{\prime}\right)=a_{R} a_{r}^{\prime} \otimes\left(b_{R}\right)_{r}, \quad \forall a, a^{\prime} \in A, b \in B, \\
& R\left(b b^{\prime} \otimes a\right)=\left(a_{R}\right)_{r} \otimes b_{R}^{\prime} b_{r}, \quad \forall a \in A_{0}, b, b^{\prime} \in B .
\end{aligned}
$$

We consider the multiplication on the vector space $A \otimes B$ uniquely defined by the formulae

$$
\begin{aligned}
& \left(1_{A} \otimes b\right)\left(a^{\prime} \otimes b^{\prime}\right)=a_{R}^{\prime} \otimes b_{R} b^{\prime}, \quad \forall a^{\prime} \in A, b, b^{\prime} \in B, \\
& (a \otimes b)\left(a^{\prime} \otimes b^{\prime}\right)=a a_{R}^{\prime} \otimes b^{\prime} b_{R}, \quad \forall a \in A_{0}, a^{\prime} \in A, b, b^{\prime} \in B .
\end{aligned}
$$

This algebra structure on $A \otimes B$ will be called an alternative twisted tensor product and will be denoted by $A \bar{\otimes}_{R} B$; the map $R$ satisfying the conditions (1.1)-(1.3) will be called an alternative twisting map. Clearly $1_{A} \otimes 1_{B}$ is the unit for $A \bar{\otimes}_{R} B$. If the algebras $A$ and $B$ are associative and $B$ is commutative then the alternative twisted tensor product $A \bar{\otimes}_{R} B$ coincides with the usual twisted tensor product $A \otimes_{R} B$ and so is an associative algebra.

Remark 1.1 If $A \bar{\otimes}_{R} B$ is an alternative twisted tensor product and $B$ is commutative, then the multiplication of $A \bar{\otimes}_{R} B$ does not depend on the decomposition $A=K \cdot 1_{A} \oplus A_{0}$.

We recall now the Cayley-Dickson process, see for instance 14. Let $B$ be an algebra and $\sigma: B \rightarrow B$ an involution, i.e. an algebra antiautomorphism with $\sigma^{2}=i d_{B}$. We fix $q \in K, q \neq 0$, 
and define $\bar{B}(q):=B \oplus B$ as vector space; we write an element of $\bar{B}(q)$ uniquely as $a+v b$, with $a, b \in B$, where, as above, $C(K, q)=K[v] /\left(v^{2}-q\right)$. Define a multiplication on $\bar{B}(q)$, by

$$
(a+v b)(c+v d)=(a c+q d \sigma(b))+v(\sigma(a) d+c b), \quad \forall a, b, c, d \in B .
$$

This algebra $\bar{B}(q)$ is said to have been obtained from $B$ by the Cayley-Dickson process.

Consider the linear map $R: B \otimes C(K, q) \rightarrow C(K, q) \otimes B$ uniquely defined by the formulae

$$
R(b \otimes 1)=1 \otimes b, \quad R(b \otimes v)=v \otimes \sigma(b), \quad \forall b \in B .
$$

Then one can check that $R$ is an alternative twisting map and we have an algebra isomorphism

$$
\bar{B}(q) \simeq C(K, q) \bar{\otimes}_{R} B, \quad a+v b \mapsto 1 \otimes a+v \otimes b, \quad \forall a, b \in B .
$$

Thus, any algebra obtained by applying the Cayley-Dickson process may be regarded as an alternative twisted tensor product of algebras. In particular, this is the case of the algebra of octonions and, by [2, of any division alternative quasialgebra.

We recall now the so-called Clifford process as introduced in [4]. Let $A$ be an algebra and $\sigma: A \rightarrow A$ an algebra automorphism which is involutive (i.e. $\sigma^{2}=i d_{A}$ ), let $q \in K, q \neq 0$ and again $C(K, q)=K[v] /\left(v^{2}-q\right)$. Define $C l(A):=A \oplus A$ as vector space and write an element of $C l(A)$ uniquely as $a+b v$, with $a, b \in A$; then $C l(A)$ becomes an algebra with multiplication given by the formula

$$
(a+b v)(c+d v)=(a c+q b \sigma(d))+(a d+b \sigma(c)) v, \quad \forall a, b, c, d \in A .
$$

This algebra $C l(A)$ is said to have been obtained from $A$ by the Clifford process.

Consider the linear map $R: C(K, q) \otimes A \rightarrow A \otimes C(K, q)$ uniquely defined by the formulae

$$
R(1 \otimes a)=a \otimes 1, \quad R(v \otimes a)=\sigma(a) \otimes v, \quad \forall a \in A .
$$

Then one can check that $R$ is an alternative twisting map and we have an algebra isomorphism

$$
C l(A) \simeq A \bar{\otimes}_{R} C(K, q), \quad a+b v \mapsto a \otimes 1+b \otimes v, \quad \forall a, b \in A .
$$

If $A$ is associative, since $C(K, q)$ is associative and commutative it follows that $A \bar{\otimes}_{R} C(K, q)$ is a usual twisted tensor product of associative algebras, so $C l(A)$ is an associative algebra (this was noted in [4] too). By [4, the usual Clifford algebras may be obtained from the field $K$ by iterating the Clifford process. Thus, the alternative twisted tensor product of algebras as introduced above provides a common generalization of Clifford algebras and Cayley algebras.

Let $A \bar{\otimes}_{R} B$ be an alternative twisted tensor product of algebras. Clearly the maps

$$
\begin{gathered}
A \rightarrow A \bar{\otimes}_{R} B, \quad a \mapsto a \otimes 1_{B}, \\
B \rightarrow A \bar{\otimes}_{R} B . \quad b \mapsto 1_{A} \otimes b,
\end{gathered}
$$

are algebra maps, and $\left(a \otimes 1_{B}\right)\left(1_{A} \otimes b\right)=a \otimes b$ in $A \bar{\otimes}_{R} B$, for all $a \in A, b \in B$. We have already seen that if $A$ is associative and $B$ is associative and commutative then $A \bar{\otimes}_{R} B$ is associative. We can prove a converse of this, generalizing the well-known fact that the algebra $\bar{B}(q)$ obtained from $B$ by the Cayley-Dickson process is associative if and only if $B$ is associative and commutative: 
Proposition 1.2 If $A_{0} \neq 0$ (i.e. $\operatorname{dim}(A) \geq 2$ ) and $A \bar{\otimes}_{R} B$ is associative, then $A$ is associative and $B$ is associative and commutative. Thus, in this case $A \bar{\otimes}_{R} B$ is a usual twisted tensor product of associative algebras.

Proof. Obviously $A$ and $B$ are associative, as they are embedded as algebras into $A \bar{\otimes}_{R} B$. Let $a \in A_{0}, a \neq 0$ and $b, b^{\prime} \in B$. We have:

$$
\begin{aligned}
& \left(a \otimes 1_{B}\right)\left[\left(1_{A} \otimes b\right)\left(1_{A} \otimes b^{\prime}\right)\right]=a \otimes b b^{\prime}, \\
& {\left[\left(a \otimes 1_{B}\right)\left(1_{A} \otimes b\right)\right]\left(1_{A} \otimes b^{\prime}\right)=(a \otimes b)\left(1_{A} \otimes b^{\prime}\right)=a \otimes b^{\prime} b,}
\end{aligned}
$$

and so the associativity of $A \bar{\otimes}_{R} B$ implies $b b^{\prime}=b^{\prime} b$.

\section{An isomorphism theorem}

It is known (see for instance [17) that the Cayley-Dickson process admits a second description, different from the one presented above, but equivalent to it, which we recall now.

We begin with an algebra $B$ and an involution $\sigma$ on it, we fix $q \in K, q \neq 0$ and consider again the algebra $C(K, q)=K[v] /\left(v^{2}-q\right)$. Define $\underline{B}(q):=B \oplus B$ as vector space, and write an element of $\underline{B}(q)$ uniquely as $a+b v$, with $a, b \in B$. Define a multiplication on $\underline{B}(q)$ by

$$
(a+b v)(c+d v)=(a c+q \sigma(d) b)+(b \sigma(c)+d a) v, \quad \forall a, b, c, d \in B .
$$

Then one can see that we have an algebra isomorphism

$$
\underline{B}(q) \simeq \bar{B}(q), \quad a+b v \mapsto a+v \sigma(b), \quad \forall a, b \in B .
$$

Our next aim is to give an interpretation of this fact in terms of alternative twisted tensor products of algebras. To begin with, we note that if $A \bar{\otimes}_{R} B$ is such an alternative twisted tensor product, the algebras $A$ and $B$ do not play a symmetric rôle in its construction. Thus, we construct first a new kind of product, a sort of mirror image of $A \bar{\otimes}_{R} B$. Namely, we begin with two algebras $C, D$ and a linear map $P: D \otimes C \rightarrow C \otimes D$, with Sweedler-type notation as before, and we assume that the following conditions are satisfied (denote by $p$ another copy of $P$ ):

$$
\begin{aligned}
& P\left(1_{D} \otimes c\right)=c \otimes 1_{D}, \quad P\left(d \otimes 1_{C}\right)=1_{C} \otimes d, \quad \forall c \in C, d \in D, \\
& P\left(d \otimes c c^{\prime}\right)=c_{p}^{\prime} c_{P} \otimes\left(d_{P}\right)_{p}, \quad \forall c, c^{\prime} \in C, d \in D_{0}, \\
& P\left(d d^{\prime} \otimes c\right)=\left(c_{P}\right)_{p} \otimes d_{p} d_{P}^{\prime}, \quad \forall c \in C, d, d^{\prime} \in D .
\end{aligned}
$$

We consider the multiplication on the vector space $C \otimes D$ uniquely defined by the formulae

$$
\begin{aligned}
& (c \otimes d)\left(c^{\prime} \otimes 1_{D}\right)=c c_{P}^{\prime} \otimes d_{P}, \quad \forall c, c^{\prime} \in C, d \in D, \\
& (c \otimes d)\left(c^{\prime} \otimes d^{\prime}\right)=c_{P}^{\prime} c \otimes d_{P} d^{\prime}, \quad \forall c, c^{\prime} \in C, d \in D, d^{\prime} \in D_{0} .
\end{aligned}
$$

This algebra structure on $C \otimes D$, whose unit is $1_{C} \otimes 1_{D}$, will be denoted by $C \underline{\otimes}_{P} D$. If the algebras $C$ and $D$ are associative and $C$ is commutative then $C \underline{\otimes}_{P} D$ coincides with the usual twisted tensor product $C \otimes_{P} D$ and so is an associative algebra.

Consider now again an algebra $B$ with an involution $\sigma$ and the linear map $P: C(K, q) \otimes B \rightarrow$ $B \otimes C(K, q)$ uniquely defined by the formulae

$$
P(1 \otimes b)=b \otimes 1, \quad P(v \otimes b)=\sigma(b) \otimes v, \quad \forall b \in B
$$


Then $P$ satisfies the conditions (2.2)-(2.4) and we have an algebra isomorphism

$$
\underline{B}(q) \simeq B \underline{\otimes}_{P} C(K, q), \quad a+b v \mapsto a \otimes 1+b \otimes v, \quad \forall a, b \in B .
$$

We can regard now the isomorphism (2.1) between the two formulations $\underline{B}(q)$ and $\bar{B}(q)$ of the Cayley-Dickson process as follows: we have an algebra isomorphism $\varphi: B \underline{\otimes}_{P} C(K, q) \simeq$ $C(K, q) \bar{\otimes}_{R} B$, given by

$$
\varphi(b \otimes 1)=1 \otimes b, \quad \varphi(b \otimes v)=v \otimes \sigma(b), \quad \forall b \in B,
$$

where $R$ is the map given by (1.4) and $P$ is the map given by (2.5).

It turns out that this result is a particular case of a general property of alternative twisted tensor products of algebras:

Theorem 2.1 Let $A \bar{\otimes}_{R} B$ be an alternative twisted tensor product of algebras such that the map $R$ is bijective, with inverse denoted by $P: A \otimes B \rightarrow B \otimes A$. Assume that the following conditions are satisfied:

$$
\begin{aligned}
& \left(i d_{A} \otimes \tau_{B, B}\right) \circ\left(R \otimes i d_{B}\right) \circ\left(i d_{B} \otimes R\right)=\left(R \otimes i d_{B}\right) \circ\left(i d_{B} \otimes R\right) \circ\left(\tau_{B, B} \otimes i d_{A}\right), \\
& R\left(B \otimes A_{0}\right)=A_{0} \otimes B .
\end{aligned}
$$

Then the map $P$ satisfies the conditions (2.2)-2.4) (for $C=B$ and $D=A$ ) and $R: B \underline{\otimes}_{P} A \rightarrow$ $A \bar{\otimes}_{R} B$ is an algebra isomorphism.

Proof. It is obvious that $P$ satisfies (2.2) because $R$ satisfies (1.1), and $P$ satisfies (2.4) because $R$ satisfies (1.2). We have to prove that $P$ satisfies (2.3). The condition (1.3) for $R$ may be written on $B \otimes B \otimes A_{0}$ as

$$
R \circ\left(\mu_{B} \otimes i d_{A}\right)=\left(i d_{A} \otimes \mu_{B}\right) \circ\left(i d_{A} \otimes \tau_{B, B}\right) \circ\left(R \otimes i d_{B}\right) \circ\left(i d_{B} \otimes R\right),
$$

which by using (2.7) becomes

$$
R \circ\left(\mu_{B} \otimes i d_{A}\right)=\left(i d_{A} \otimes \mu_{B}\right) \circ\left(R \otimes i d_{B}\right) \circ\left(i d_{B} \otimes R\right) \circ\left(\tau_{B, B} \otimes i d_{A}\right),
$$

which by composing with the appropriate maps and using (2.8) becomes

$$
P \circ\left(i d_{A} \otimes \mu_{B}\right)=\left(\mu_{B} \otimes i d_{A}\right) \circ\left(\tau_{B, B} \otimes i d_{A}\right) \circ\left(i d_{B} \otimes P\right) \circ\left(P \otimes i d_{B}\right)
$$

as an equality of maps from $A_{0} \otimes B \otimes B$ to $B \otimes A_{0}$, and this is exactly (2.3).

The only thing left to prove is that $R$ is multiplicative. First, it is obvious that

$$
R\left(\left(b \otimes 1_{A}\right)\left(b^{\prime} \otimes 1_{A}\right)\right)=R\left(b \otimes 1_{A}\right) R\left(b^{\prime} \otimes 1_{A}\right), \quad \forall b, b^{\prime} \in B .
$$

Take now $a \in A_{0}, b, b^{\prime} \in B$. By making use of (2.8), we compute:

$$
\begin{array}{rll}
R\left((b \otimes a)\left(b^{\prime} \otimes 1_{A}\right)\right) & = & R\left(b b_{P}^{\prime} \otimes a_{P}\right) \\
& \stackrel{\sqrt[1.3]{=}}{=} & \left(\left(a_{P}\right)_{R}\right)_{r} \otimes\left(b_{P}^{\prime}\right)_{R} b_{r} \\
\stackrel{P=R^{-1}}{=} & a_{r} \otimes b^{\prime} b_{r} \\
= & \left(a_{r} \otimes b_{r}\right)\left(1_{A} \otimes b^{\prime}\right) \\
= & R(b \otimes a) R\left(b^{\prime} \otimes 1_{A}\right) .
\end{array}
$$


Take now $a^{\prime} \in A_{0}, b, b^{\prime} \in B$. We compute:

$$
\begin{array}{rll}
R\left(\left(b \otimes 1_{A}\right)\left(b^{\prime} \otimes a^{\prime}\right)\right) & = & R\left(b^{\prime} b \otimes a^{\prime}\right) \\
& \stackrel{\sqrt[1.3]{=}}{=}\left(a_{R}^{\prime}\right)_{r} \otimes b_{R} b_{r}^{\prime} \\
& \stackrel{2.7}{=} & \left(a_{R}^{\prime}\right)_{r} \otimes b_{r} b_{R}^{\prime} \\
= & \left(1_{A} \otimes b\right)\left(a_{R}^{\prime} \otimes b_{R}^{\prime}\right) \\
= & R\left(b \otimes 1_{A}\right) R\left(b^{\prime} \otimes a^{\prime}\right) .
\end{array}
$$

Finally, take $a, a^{\prime} \in A_{0}, b, b^{\prime} \in B$. Using again (2.8), we compute (denote $r, \mathcal{R}$ two copies of $R$ ):

$$
\begin{array}{cll}
R\left((b \otimes a)\left(b^{\prime} \otimes a^{\prime}\right)\right) & = & R\left(b_{P}^{\prime} b \otimes a_{P} a^{\prime}\right) \\
& \stackrel{1.2}{=} & \left(a_{P}\right)_{R} a_{r}^{\prime} \otimes\left(\left(b_{P}^{\prime} b\right)_{R}\right)_{r} \\
& \stackrel{1.3}{=} & \left(\left(a_{P}\right)_{\mathcal{R}}\right)_{R} a_{r}^{\prime} \otimes\left(b_{\mathcal{R}}\left(b_{P}^{\prime}\right)_{R}\right)_{r} \\
& \stackrel{1.7}{=} & \left(\left(a_{P}\right)_{\mathcal{R}}\right)_{R} a_{r}^{\prime} \otimes\left(b_{R}\left(b_{P}^{\prime}\right)_{\mathcal{R}}\right)_{r} \\
P=R^{-1} & a_{R} a_{r}^{\prime} \otimes\left(b_{R} b^{\prime}\right)_{r} \\
\stackrel{1.3}{=} & a_{R}\left(a_{r}^{\prime}\right)_{\mathcal{R}} \otimes b_{r}^{\prime}\left(b_{R}\right)_{\mathcal{R}} \\
= & \left(a_{R} \otimes b_{R}\right)\left(a_{r}^{\prime} \otimes b_{r}^{\prime}\right) \\
= & R(b \otimes a) R\left(b^{\prime} \otimes a^{\prime}\right),
\end{array}
$$

showing that indeed $R$ is multiplicative.

If we consider again an algebra $B$ with an involution $\sigma$, it is easy to see that the map $R$ given by (1.4) satisfies the hypotheses of Theorem 2.1, and the isomorphism $B \underline{\otimes}_{P} C(K, q) \simeq$ $C(K, q) \bar{\otimes}_{R} B$ provided by the Theorem coincides with the isomorphism $\varphi$ given by (2.6).

Let us also note that the condition (2.7) is a particular case of the hexagon (or braid) relation which arose in [11] in the context of iterated twisted tensor products of associative algebras.

\section{Lifting involutions to alternative twisted tensor products}

Let $B$ be an algebra and $\sigma: B \rightarrow B$ an involution. It is well-known (see [14]) that the map

$$
\bar{\sigma}: \bar{B}(q) \rightarrow \bar{B}(q), \quad \bar{\sigma}(a+v b)=\sigma(a)-v b, \quad \forall a, b \in B,
$$

is an involution for the Cayley-Dickson algebra $\bar{B}(q)$. We will show that this fact is a particular case of a general result about alternative twisted tensor products of algebras, which in turn is analogous to a result in [16] about twisted tensor products of associative algebras:

Theorem 3.1 Let $A \bar{\otimes}_{R} B$ be an alternative twisted tensor product of algebras, $\sigma_{A}: A \rightarrow A$ and $\sigma_{B}: B \rightarrow B$ two involutions, and define $\bar{\sigma}: A \otimes B \rightarrow A \otimes B, \bar{\sigma}:=R \circ\left(\sigma_{B} \otimes \sigma_{A}\right) \circ \tau_{A, B}$. Assume that $R$ satisfies (2.7) and moreover the following conditions hold:

$$
\begin{aligned}
& R\left(B \otimes A_{0}\right) \subseteq A_{0} \otimes B, \\
& \sigma_{A}\left(A_{0}\right) \subseteq A_{0}, \\
& \bar{\sigma}^{2}=i d_{A \otimes B} .
\end{aligned}
$$

Then $\bar{\sigma}$ is an involution for $A \bar{\otimes}_{R} B$. 
Proof. Note first that $\bar{\sigma}$ is given by the formula $\bar{\sigma}(a \otimes b)=\sigma_{A}(a)_{R} \otimes \sigma_{B}(b)_{R}$, for all $a \in A$, $b \in B$, and (3.4) together with the fact that $\sigma_{A}$ and $\sigma_{B}$ are involutions imply

$$
\sigma_{A}\left(a_{R}\right)_{r} \otimes \sigma_{B}\left(b_{R}\right)_{r}=\sigma_{A}(a) \otimes \sigma_{B}(b), \quad \forall a \in A, b \in B .
$$

In view of (3.4), the only thing we need to prove is that $\bar{\sigma}$ is antimultiplicative. We will denote by $r, \mathcal{R}, \bar{R}$ some more copies of $R$. First, it is easy to see that

$$
\bar{\sigma}\left(\left(1_{A} \otimes b\right)\left(1_{A} \otimes b^{\prime}\right)\right)=\bar{\sigma}\left(1_{A} \otimes b^{\prime}\right) \bar{\sigma}\left(1_{A} \otimes b\right), \quad \forall b, b^{\prime} \in B .
$$

Take now $a^{\prime} \in A_{0}, b, b^{\prime} \in B$. By using (3.2) and (3.3), we compute:

$$
\begin{aligned}
& \bar{\sigma}\left(\left(1_{A} \otimes b\right)\left(a^{\prime} \otimes b^{\prime}\right)\right)=\bar{\sigma}\left(a_{R}^{\prime} \otimes b_{R} b^{\prime}\right) \\
& =\sigma_{A}\left(a_{R}^{\prime}\right)_{r} \otimes \sigma_{B}\left(b_{R} b^{\prime}\right)_{r} \\
& =\sigma_{A}\left(a_{R}^{\prime}\right)_{r} \otimes\left(\sigma_{B}\left(b^{\prime}\right) \sigma_{B}\left(b_{R}\right)\right)_{r} \\
& \stackrel{1.3}{=}\left(\sigma_{A}\left(a_{R}^{\prime}\right)_{\mathcal{R}}\right)_{r} \otimes \sigma_{B}\left(b_{R}\right)_{\mathcal{R}} \sigma_{B}\left(b^{\prime}\right)_{r} \\
& \stackrel{3.5}{=} \sigma_{A}\left(a^{\prime}\right)_{r} \otimes \sigma_{B}(b) \sigma_{B}\left(b^{\prime}\right)_{r} \\
& =\left(\sigma_{A}\left(a^{\prime}\right)_{r} \otimes \sigma_{B}\left(b^{\prime}\right)_{r}\right)\left(1_{A} \otimes \sigma_{B}(b)\right) \\
& =\bar{\sigma}\left(a^{\prime} \otimes b^{\prime}\right) \bar{\sigma}\left(1_{A} \otimes b\right) .
\end{aligned}
$$

Take now $a \in A_{0}, b, b^{\prime} \in B$; by using (3.3), we compute:

$$
\begin{aligned}
& \bar{\sigma}\left((a \otimes b)\left(1_{A} \otimes b^{\prime}\right)\right)=\bar{\sigma}\left(a \otimes b^{\prime} b\right) \\
& =\sigma_{A}(a)_{R} \otimes \sigma_{B}\left(b^{\prime} b\right)_{R} \\
& =\sigma_{A}(a)_{R} \otimes\left(\sigma_{B}(b) \sigma_{B}\left(b^{\prime}\right)\right)_{R} \\
& \stackrel{1.3}{=}\left(\sigma_{A}(a)_{R}\right)_{r} \otimes \sigma_{B}\left(b^{\prime}\right)_{R} \sigma_{B}(b)_{r} \\
& \stackrel{2.7}{=}\left(\sigma_{A}(a)_{R}\right)_{r} \otimes \sigma_{B}\left(b^{\prime}\right)_{r} \sigma_{B}(b)_{R} \\
& =\left(1_{A} \otimes \sigma_{B}\left(b^{\prime}\right)\right)\left(\sigma_{A}(a)_{R} \otimes \sigma_{B}(b)_{R}\right) \\
& =\bar{\sigma}\left(1_{A} \otimes b^{\prime}\right) \bar{\sigma}(a \otimes b) .
\end{aligned}
$$

Finally, take $a, a^{\prime} \in A_{0}, b, b^{\prime} \in B$. Again by using (3.2) and (3.3) we compute:

$$
\begin{aligned}
& \bar{\sigma}\left((a \otimes b)\left(a^{\prime} \otimes b^{\prime}\right)\right)=\bar{\sigma}\left(a a_{R}^{\prime} \otimes b^{\prime} b_{R}\right) \\
& =\sigma_{A}\left(a a_{R}^{\prime}\right)_{r} \otimes \sigma_{B}\left(b^{\prime} b_{R}\right)_{r} \\
& =\left(\sigma_{A}\left(a_{R}^{\prime}\right) \sigma_{A}(a)\right)_{r} \otimes\left(\sigma_{B}\left(b_{R}\right) \sigma_{B}\left(b^{\prime}\right)\right)_{r} \\
& \stackrel{1.2}{=} \sigma_{A}\left(a_{R}^{\prime}\right)_{\mathcal{R}} \sigma_{A}(a)_{r} \otimes\left(\left(\sigma_{B}\left(b_{R}\right) \sigma_{B}\left(b^{\prime}\right)\right)_{\mathcal{R}}\right)_{r} \\
& \stackrel{1.30}{=}\left(\sigma_{A}\left(a_{R}^{\prime}\right)_{\bar{R}}\right)_{\mathcal{R}} \sigma_{A}(a)_{r} \otimes\left(\sigma_{B}\left(b^{\prime}\right)_{\bar{R}} \sigma_{B}\left(b_{R}\right)_{\mathcal{R}}\right)_{r} \\
& \stackrel{2.7}{=}\left(\sigma_{A}\left(a_{R}^{\prime}\right)_{\bar{R}}\right)_{\mathcal{R}} \sigma_{A}(a)_{r} \otimes\left(\sigma_{B}\left(b^{\prime}\right)_{\mathcal{R}} \sigma_{B}\left(b_{R}\right)_{\bar{R}}\right)_{r} \\
& \stackrel{\text { 3.5 }}{=} \sigma_{A}\left(a^{\prime}\right)_{\mathcal{R}} \sigma_{A}(a)_{r} \otimes\left(\sigma_{B}\left(b^{\prime}\right)_{\mathcal{R}} \sigma_{B}(b)\right)_{r} \\
& \stackrel{1.3}{=} \sigma_{A}\left(a^{\prime}\right)_{\mathcal{R}}\left(\sigma_{A}(a)_{R}\right)_{r} \otimes \sigma_{B}(b)_{R}\left(\sigma_{B}\left(b^{\prime}\right)_{\mathcal{R}}\right)_{r} \\
& =\left(\sigma_{A}\left(a^{\prime}\right)_{\mathcal{R}} \otimes \sigma_{B}\left(b^{\prime}\right)_{\mathcal{R}}\right)\left(\sigma_{A}(a)_{R} \otimes \sigma_{B}(b)_{R}\right) \\
& =\bar{\sigma}\left(a^{\prime} \otimes b^{\prime}\right) \bar{\sigma}(a \otimes b) \text {, }
\end{aligned}
$$

finishing the proof. 
Remark 3.2 There exists at least one natural case when (3.4) holds automatically. Namely, let $A, B$ be algebras with $B$ commutative; then obviously the flip map $\tau_{A, B}$ is an alternative twisting map. If $\sigma_{A}: A \rightarrow A$ and $\sigma_{B}: B \rightarrow B$ are two involutions, then obviously $\bar{\sigma}$ is given by $\bar{\sigma}(a \otimes b)=\sigma_{A}(a) \otimes \sigma_{B}(b)$, for all $a \in A, b \in B$, and it clearly satisfies (3.4).

Consider now the description $\bar{B}(q) \simeq C(K, q) \bar{\otimes}_{R} B$ of the Cayley-Dickson process as an alternative twisted tensor product. We denote $\sigma_{B}=\sigma, A=C(K, q)$, and consider the involution $\sigma_{A}$ of $A$ given by $\sigma_{A}(1)=1, \sigma_{A}(v)=-v$. Then one can easily check that the hypotheses of Theorem 3.1 are fulfilled, and the involution $\bar{\sigma}$ on $A \bar{\otimes}_{R} B$ provided by the Theorem coincides with the involution (3.1) via the identification $\bar{B}(q) \simeq C(K, q) \bar{\otimes}_{R} B$.

We recall from [4] that if $A$ is an algebra and $\sigma: A \rightarrow A$ is an involutive automorphism, then $\sigma$ can be lifted to an involutive automorphism $\bar{\sigma}: C l(A) \rightarrow C l(A), \bar{\sigma}(a+b v)=\sigma(a)-\sigma(b) v$, for all $a, b \in A$. It is easy to see that this fact is a particular case of the following result about alternative twisted tensor products, which in turn is the analogue of a result in [7] for twisted tensor products of associative algebras:

Proposition 3.3 Let $A \bar{\otimes}_{R} B$ and $E \bar{\otimes}_{T} F$ be two alternative twisted tensor products of algebras and $f: A \rightarrow E, g: B \rightarrow F$ two algebra maps such that $f\left(A_{0}\right) \subseteq E_{0}$ and $(f \otimes g) \circ R=T \circ(g \otimes f)$. Then $f \otimes g: A \bar{\otimes}_{R} B \rightarrow E \bar{\otimes}_{T} F$ is an algebra map.

Proof. A straightforward computation.

\section{A class of examples}

We recall that an algebra $D$ is called alternative if $(x x) y=x(x y)$ and $x(y y)=(x y) y$, for all $x, y \in D$. These identities are called the left and respectively right alternative laws. We also recall that an involution $\sigma$ on an algebra $B$ is called strong if $b+\sigma(b) \in K \cdot 1_{B}$ and $b \sigma(b) \in K \cdot 1_{B}$ for all $b \in B$. In this case we denote as usual $b+\sigma(b)=t(b) 1_{B}$ and $b \sigma(b)(=\sigma(b) b)=n(b) 1_{B}$. The maps $t, n: B \rightarrow K$ are called the trace and respectively norm of $B$.

Proposition 4.1 (i) Let $A=K \cdot 1_{A} \oplus A_{0}$ be an algebra satisfying the condition

$$
A_{0} \cdot A_{0} \subseteq K \cdot 1_{A},
$$

let $B$ be an algebra and $\sigma: B \rightarrow B$ an involution. Then the map $R: B \otimes A \rightarrow A \otimes B$ defined by

$$
R\left(b \otimes 1_{A}\right)=1_{A} \otimes b, \quad R(b \otimes a)=a \otimes \sigma(b), \quad \forall a \in A_{0}, b \in B,
$$

is an alternative twisting map.

(ii) If moreover $A$ and $B$ are alternative and $\sigma$ is strong, then, for all $b, b^{\prime} \in B$ and all "homogeneous" $a, a^{\prime} \in A$ (i.e. a and $a^{\prime}$ belong either to $K \cdot 1_{A}$ or to $A_{0}$ ), the left and right alternative laws for tensor monomials hold:

$$
\begin{aligned}
& {[(a \otimes b)(a \otimes b)]\left(a^{\prime} \otimes b^{\prime}\right)=(a \otimes b)\left[(a \otimes b)\left(a^{\prime} \otimes b^{\prime}\right)\right],} \\
& (a \otimes b)\left[\left(a^{\prime} \otimes b^{\prime}\right)\left(a^{\prime} \otimes b^{\prime}\right)\right]=\left[(a \otimes b)\left(a^{\prime} \otimes b^{\prime}\right)\right]\left(a^{\prime} \otimes b^{\prime}\right) .
\end{aligned}
$$

Proof. (i) Follows by a direct computation; note that (4.1) is used for proving (1.2).

(ii) We check the left alternative law, while the proof of the right alternative law is similar and left to the reader. Note first that the alternativity of $B$ implies

$$
\left(b^{\prime} \sigma(b)\right) b=n(b) b^{\prime}
$$




$$
\left(b^{\prime} b\right) \sigma(b)=n(b) b^{\prime},
$$

for all $b, b^{\prime} \in B$. Indeed, we have

$$
\begin{aligned}
\left(b^{\prime} \sigma(b)\right) b & =\left(b^{\prime}\left(t(b) 1_{B}-b\right)\right) b \\
& =t(b) b^{\prime} b-\left(b^{\prime} b\right) b \\
& =t(b) b^{\prime} b-b^{\prime}(b b) \\
& =b^{\prime}(t(b) b-b b) \\
& =b^{\prime}(\sigma(b) b) \\
& =n(b) b^{\prime}
\end{aligned}
$$

and similarly for (4.5).

We have to prove that (4.2) holds for all homogeneous $a, a^{\prime} \in A$ and all $b, b^{\prime} \in B$. This is obvious if $a=a^{\prime}=1_{A}$, so we only have three cases to analyze:

case 1: $a, a^{\prime} \in A_{0}$; we compute:

$$
\begin{array}{rll}
{[(a \otimes b)(a \otimes b)]\left(a^{\prime} \otimes b^{\prime}\right)} & = & \left(a^{2} \otimes b \sigma(b)\right)\left(a^{\prime} \otimes b^{\prime}\right) \\
= & \left(a^{2} \otimes n(b) 1_{B}\right)\left(a^{\prime} \otimes b^{\prime}\right) \\
& \stackrel{4.1}{=} & a^{2} a^{\prime} \otimes n(b) b^{\prime}, \\
(a \otimes b)\left[(a \otimes b)\left(a^{\prime} \otimes b^{\prime}\right)\right] & = & (a \otimes b)\left(a a^{\prime} \otimes b^{\prime} \sigma(b)\right) \\
& \stackrel{4.1}{=} & a\left(a a^{\prime}\right) \otimes\left(b^{\prime} \sigma(b)\right) b \\
& \stackrel{4.4}{=} & a^{2} a^{\prime} \otimes n(b) b^{\prime} .
\end{array}
$$

case 2: $a \in A_{0}, a^{\prime}=1_{A}$; we compute:

$$
\begin{aligned}
{[(a \otimes b)(a \otimes b)]\left(1_{A} \otimes b^{\prime}\right)=} & \left(a^{2} \otimes n(b) 1_{B}\right)\left(1_{A} \otimes b^{\prime}\right) \\
= & a^{2} \otimes n(b) b^{\prime} \\
(a \otimes b)\left[(a \otimes b)\left(1_{A} \otimes b^{\prime}\right)\right] & =(a \otimes b)\left(a \otimes b^{\prime} b\right) \\
& =a^{2} \otimes\left(b^{\prime} b\right) \sigma(b) \\
& \stackrel{4.5}{=} a^{2} \otimes n(b) b^{\prime} .
\end{aligned}
$$

case 3: $a=1_{A}, a^{\prime} \in A_{0}$; we compute:

$$
\begin{aligned}
{\left[\left(1_{A} \otimes b\right)\left(1_{A} \otimes b\right)\right]\left(a^{\prime} \otimes b^{\prime}\right) } & =\left(1_{A} \otimes b^{2}\right)\left(a^{\prime} \otimes b^{\prime}\right) \\
& =a^{\prime} \otimes \sigma\left(b^{2}\right) b^{\prime} \\
& =a^{\prime} \otimes \sigma(b)^{2} b^{\prime} \\
\left(1_{A} \otimes b\right)\left[\left(1_{A} \otimes b\right)\left(a^{\prime} \otimes b^{\prime}\right)\right] & =\left(1_{A} \otimes b\right)\left(a^{\prime} \otimes \sigma(b) b^{\prime}\right) \\
& =a^{\prime} \otimes \sigma(b)\left(\sigma(b) b^{\prime}\right) \\
& =a^{\prime} \otimes \sigma(b)^{2} b^{\prime},
\end{aligned}
$$

finishing the proof. 
Remark 4.2 Although the alternative laws for homogeneous tensor monomials hold, in general this does not imply that $A \bar{\otimes}_{R} B$ is an alternative algebra. A concrete counterexample is provided by the algebra of sedenions, which is not alternative although is an alternative twisted tensor product between $C(K, q)$ and the (alternative) algebra of octonions.

Let now $B$ be an algebra endowed with a strong involution $\sigma: B \rightarrow B$, let $q, r \in K \backslash\{0\}$ and consider the 3 -dimensional algebra $A$ over $K$ with basis $\{1, v, z\}$, where 1 is the unit and $v^{2}=q 1, z^{2}=r 1, v z=z v=0$. Note that $A$ is not associative, not even alternative (as $q z=(v v) z \neq v(v z)=0)$. If we define $A_{0}=K v \oplus K z$, then obviously we have $A_{0} \cdot A_{0} \subseteq K \cdot 1_{A}$, so we can define the alternative twisting map $R: B \otimes A \rightarrow A \otimes B, R\left(b \otimes 1_{A}\right)=1_{A} \otimes b$, $R(b \otimes v)=v \otimes \sigma(b), R(b \otimes z)=z \otimes \sigma(b)$, for all $b \in B$. We denote the algebra $A \bar{\otimes}_{R} B$ by $\bar{B}(q, r)$ and we write an element of this algebra uniquely as $x=a+v b+z c$, with $a, b, c \in B$. Then $\operatorname{dim}(\bar{B}(q, r))=3 \cdot \operatorname{dim}(B)$ and the multiplication in $\bar{B}(q, r)$ is given by

$$
(a+v b+z c)\left(a^{\prime}+v b^{\prime}+z c^{\prime}\right)=\left(a a^{\prime}+q b^{\prime} \sigma(b)+r c^{\prime} \sigma(c)\right)+v\left(\sigma(a) b^{\prime}+a^{\prime} b\right)+z\left(\sigma(a) c^{\prime}+a^{\prime} c\right) .
$$

Proposition 4.3 The map $\bar{\sigma}: \bar{B}(q, r) \rightarrow \bar{B}(q, r), \bar{\sigma}(a+v b+z c):=\sigma(a)-v b-z c$, for all $a, b, c \in B$, is a strong involution for $\bar{B}(q, r)$. Moreover, the trace and norm of the element $x=a+v b+z c \in \bar{B}(q, r)$ are given by $t(x)=t(a)$ and $n(x)=n(a)-q n(b)-r n(c)$, where $t$ and $n$ are the trace and norm on $B$.

Proof. The fact that $\bar{\sigma}$ is an involution follows either by a direct computation or as a consequence of Theorem 3.1, and the fact that $\bar{\sigma}$ is strong, together with the explicit formulae for the trace and the norm, follow easily by direct computation.

Remark 4.4 Obviously the Cayley-Dickson algebras $\bar{B}(q)$ and $\bar{B}(r)$ are subalgebras in $\bar{B}(q, r)$. On the other hand, if we consider the algebra $\overline{\bar{B}(q)}(r)$ obtained by applying the Cayley-Dickson process to $\bar{B}(q)$, it might be tempting to believe that $\bar{B}(q, r)$ is a subalgebra in $\overline{\bar{B}}(q)(r)$, but this is not true, since $v z=0$ in $\bar{B}(q, r)$ while $v z \neq 0$ in $\overline{\bar{B}(q)}(r)$.

Let $B$ be an associative algebra and $\sigma: B \rightarrow B$ a strong involution. It is well-known that in this case the Cayley-Dickson algebra $\bar{B}(q)$ is alternative. On the other hand, since the 3-dimensional algebra $A$ defined above is a subalgebra in $\bar{B}(q, r)$ and $A$ is not alternative, it follows that $\bar{B}(q, r)$ is never alternative. However, since $\bar{B}(q, r)$ is endowed with a strong involution (hence is of degree two), it follows that it is always power-associative.

We will see that the algebras $\bar{B}(q, r)$ share some more common properties with the CayleyDickson algebras $\bar{B}(q)$. We recall that an algebra $D$ is called flexible if $(x y) x=x(y x)$ for all $x, y \in D$. By [15] we know that all Cayley-Dickson algebras are flexible. We will prove that a similar result holds for the algebras $\bar{B}(q, r)$. We need to recall some formulae from [15], which are valid for any flexible algebra $B$ endowed with a strong involution $\sigma$ :

$$
\begin{aligned}
& (x y) \sigma(y)=\sigma(y)(y x)=y(\sigma(y) x)=(x \sigma(y)) y, \\
& (u \sigma(y)) x+y(\sigma(u) x)=x(y \sigma(u))+(x u) \sigma(y),
\end{aligned}
$$

for all $x, y, u \in B$. Also, by writing $\sigma(x)=t(x) 1_{B}-x$, one can easily check the following formula:

$$
(\sigma(u) \sigma(x)) y+x(u y)=\sigma(x)(\sigma(u) y)+(u x) y .
$$


Proposition 4.5 Let $B$ be an algebra and $\sigma: B \rightarrow B$ a strong involution. Then $\bar{B}(q, r)$ is flexible if and only if $B$ is flexible.

Proof. If $\bar{B}(q, r)$ is flexible then obviously $B$ is flexible, as it is a subalgebra of $\bar{B}(q, r)$. Conversely, assume that $B$ is flexible and let $X=a+v b+z c$ and $Y=a^{\prime}+v b^{\prime}+z c^{\prime}$ be two elements in $\bar{B}(q, r)$. We can easily compute:

$$
\begin{aligned}
(X Y) X= & \left(a a^{\prime}\right) a+q\left(\left(b^{\prime} \sigma(b)\right) a+b\left(\sigma\left(b^{\prime}\right) a\right)\right)+r\left(\left(c^{\prime} \sigma(c)\right) a+c\left(\sigma\left(c^{\prime}\right) a\right)\right) \\
& +q b\left(\sigma(b) \sigma\left(a^{\prime}\right)\right)+r c\left(\sigma(c) \sigma\left(a^{\prime}\right)\right) \\
& +v\left(\left(\sigma\left(a^{\prime}\right) \sigma(a)\right) b+a\left(a^{\prime} b\right)+q\left(b \sigma\left(b^{\prime}\right)\right) b+r\left(c \sigma\left(c^{\prime}\right)\right) b+a\left(\sigma(a) b^{\prime}\right)\right) \\
& +z\left(\left(\sigma\left(a^{\prime}\right) \sigma(a)\right) c+a\left(a^{\prime} c\right)+q\left(b \sigma\left(b^{\prime}\right)\right) c+r\left(c \sigma\left(c^{\prime}\right)\right) c+a\left(\sigma(a) c^{\prime}\right)\right), \\
X(Y X)= & a\left(a^{\prime} a\right)+q\left(a\left(b \sigma\left(b^{\prime}\right)\right)+\left(a b^{\prime}\right) \sigma(b)\right)+r\left(a\left(c \sigma\left(c^{\prime}\right)\right)+\left(a c^{\prime}\right) \sigma(c)\right) \\
& +q\left(\sigma\left(a^{\prime}\right) b\right) \sigma(b)+r\left(\sigma\left(a^{\prime}\right) c\right) \sigma(c) \\
& +v\left(\sigma(a)\left(\sigma\left(a^{\prime}\right) b\right)+\left(a^{\prime} a\right) b+q\left(b \sigma\left(b^{\prime}\right)\right) b+r\left(c \sigma\left(c^{\prime}\right)\right) b+\sigma(a)\left(a b^{\prime}\right)\right) \\
& +z\left(\sigma(a)\left(\sigma\left(a^{\prime}\right) c\right)+\left(a^{\prime} a\right) c+q\left(b \sigma\left(b^{\prime}\right)\right) c+r\left(c \sigma\left(c^{\prime}\right)\right) c+\sigma(a)\left(a c^{\prime}\right)\right),
\end{aligned}
$$

and we immediately obtain $(X Y) X=X(Y X)$ by using the flexibility of $B$ together with (4.6), (4.7), (4.8).

Remark 4.6 In particular, if we take $B$ to be an associative or alternative algebra with a strong involution, we obtain an infinite family of flexible algebras, of dimensions $3^{n} \cdot \operatorname{dim}(B)$, for all positive integers $n$.

Assume now $\operatorname{char}(K) \neq 2$ and let $B$ be an algebra endowed with a strong involution $\sigma$. We recall that the norm $n$ on $B$ is called nondegenerate if the associated symmetric bilinear form

$$
(x, y)=\frac{1}{2}(n(x+y)-n(x)-n(y)), \quad \forall x, y \in B,
$$

is nondegenerate (i.e. if $(x, y)=0$ for all $y \in B$ then $x=0$ ). It is well-known (see [14], p. 70) that all Cayley-Dickson algebras have nondegenerate norms. We prove a similar result for the algebras $\bar{B}(q, r)$ :

Proposition 4.7 If $B$ has nondegenerate norm, then $\bar{B}(q, r)$ has also nondegenerate norm.

Proof. Let $x=a+v b+z c \in \bar{B}(q, r)$ such that $(x, y)=0$ for all $y=a^{\prime}+v b^{\prime}+z c^{\prime} \in \bar{B}(q, r)$. It is easy to see that

$$
\begin{aligned}
(x, y)= & \frac{1}{2}(n(x+y)-n(x)-n(y)) \\
= & \frac{1}{2}\left(n\left(a+a^{\prime}\right)-q n\left(b+b^{\prime}\right)-r n\left(c+c^{\prime}\right)-n(a)+q n(b)+r n(c)\right. \\
& \left.-n\left(a^{\prime}\right)+q n\left(b^{\prime}\right)+r n\left(c^{\prime}\right)\right) \\
= & \left(a, a^{\prime}\right)-q\left(b, b^{\prime}\right)-r\left(c, c^{\prime}\right),
\end{aligned}
$$

thus $(x, y)=0$ for all $y$ implies $\left(a, a^{\prime}\right)=q\left(b, b^{\prime}\right)+r\left(c, c^{\prime}\right)$ for all $a^{\prime}, b^{\prime}, c^{\prime} \in B$. By taking $b^{\prime}=c^{\prime}=0$ we obtain $\left(a, a^{\prime}\right)=0$ for all $a^{\prime} \in B$, and since the norm on $B$ is nondegenerate this implies $a=0$. Similarly, by taking $a^{\prime}=c^{\prime}=0$ and then $a^{\prime}=b^{\prime}=0$ and using the fact that $q \neq 0, r \neq 0$ and again the nondegeneracy of the norm on $B$, we obtain $b=0$ and respectively $c=0$; that is, $x=0$.

\section{ACKNOWLEDGEMENTS}

We would like to thank the referee for some useful comments and suggestions. 


\section{References}

[1] S. E. Akrami and S. Majid, Braided cyclic cocycles and nonassociative geometry, J. Math. Phys. 45 (2004), 3883-3911.

[2] H. Albuquerque, A. Elduque and J. M. Pérez-Izquierdo, Alternative quasialgebras, Bull. Austral. Math. Soc. 63 (2001), 257-268.

[3] H. Albuquerque and S. Majid, Quasialgebra structure of the octonions, J. Algebra 220 (1999), 188-224.

[4] H. Albuquerque and S. Majid, Clifford algebras obtained by twisting of group algebras, J. Pure Appl. Algebra 171 (2002), 133-148.

[5] H. Albuquerque and F. Panaite, On quasi-Hopf smash products and twisted tensor products of quasialgebras, Algebr. Represent. Theory 12 (2009), 199-234.

[6] E. J. Beggs and S. Majid, Quantization by cochain twists and nonassociative differentials, arXiv:math.QA/0506450.

[7] A. Borowiec and W. Marcinek, On crossed product of algebras, J. Math. Phys. 41 (2000), 6959-6975.

[8] D. Bulacu, F. Panaite, F. Van Oystaeyen, Generalized diagonal crossed products and smash products for quasi-Hopf algebras. Applications, Comm. Math. Phys. 266 (2006), 355-399.

[9] A. Cap, H. Schichl and J. Vanžura, On twisted tensor products of algebras, Comm. Algebra 23 (1995), 4701-4735.

[10] F. Hausser, F. Nill, Diagonal crossed products by duals of quasi-quantum groups, Rev. Math. Phys. 11 (1999), 553-629.

[11] P. Jara Martínez, J. López Peña, F. Panaite and F. Van Oystaeyen, On iterated twisted tensor products of algebras, Internat. J. Math. 19 (2008), 1053-1101.

[12] J. López Peña, F. Panaite and F. Van Oystaeyen, General twisting of algebras, Adv. Math. 212 (2007), 315-337.

[13] S. Majid, Gauge theory on nonassociative spaces, J. Math. Phys. 46 (2005), 103519, 23 pp.

[14] R. D. Schafer, An introduction to nonassociative algebras, Academic Press, New York and London, 1966.

[15] R. D. Schafer, On the algebras formed by the Cayley-Dickson process, Amer. J. Math. 76 (1954), 435-446.

[16] A. Van Daele and S. Van Keer, The Yang-Baxter and pentagon equation, Compositio Math. 91 (1994), 201-221.

[17] G. P. Wene, A construction relating Clifford algebras and Cayley-Dickson algebras, J. Math. Phys. 25 (1984), 2351-2353. 